\title{
Nondipole effects in interference patterns of a two-electron wave
}

\author{
Hao Liang $\odot,{ }^{1}$ Sven Grundmann $\odot,{ }^{2}$ Yong-Kang Fang $\odot,{ }^{1}$ Lei Geng $\odot,{ }^{1}$ Qihuang Gong, ${ }^{1,3,4,5}$ and Liang-You Peng $\odot{ }^{1,3,4,5, *}$ \\ ${ }^{1}$ State Key Laboratory for Mesoscopic Physics and Frontiers Science Center for Nano-optoelectronics, \\ School of Physics, Peking University, 100871 Beijing, China \\ ${ }^{2}$ Institut für Kernphysik, Goethe-Universität, Max-von-Laue-Strasse 1, D-60438 Frankfurt, Germany \\ ${ }^{3}$ Collaborative Innovation Center of Quantum Matter, Beijing 100871, China \\ ${ }^{4}$ Collaborative Innovation Center of Extreme Optics, Shanxi University, Taiyuan 030006, China \\ ${ }^{5}$ Beijing Academy of Quantum Information Sciences, Beijing 100193, China
}

(Received 1 February 2021; revised 28 April 2021; accepted 30 July 2021; published 18 August 2021)

\begin{abstract}
When the photoionization is described beyond the dipole approximation, the electron in atoms and molecules will experience a spatially dependent light phase. As demonstrated in a recent experiment on one-photon double ionization of $\mathrm{H}_{2}$ [S. Grundmann et al., Science 370, 339 (2020)], the resulting phase difference modifies the electron interference pattern. By solving the full-dimensional time-dependent Schrödinger equation beyond the dipole approximation, the present work provides corresponding ab initio calculations that have been missing so far, to the best of our knowledge. Due to the two-electron nature of this process, one may wonder about the roles played by the electron-electron correlation in the shifting of the interference fringes. Indeed, we show that the modification of the interference pattern occurs at the two-electron level, which is independent of the energy partition between the two electrons. Our numerical results agree excellently with the prediction of a simple model, in which the entangled electron pair is launched by light with a spatially dependent phase.
\end{abstract}

DOI: 10.1103/PhysRevA.104.L021101

As the simplest diatomic homonuclear molecule, the hydrogen molecule $\left(\mathrm{H}_{2}\right)$ provides a natural setup for a double-slit interference experiment [1]. Electrons emitted from the two nuclei carry phases with a difference of $\mathbf{p}_{e} \cdot \mathbf{R}$, which leads to the oscillating behavior in the electron angular distribution [2-4]. Here, $\mathbf{p}_{e}$ is the momentum vector of the electron and $\mathbf{R}$ is the internuclear vector. In most experiments, double ionization is used for the analysis: Both protons are measured in coincidence with the electrons to determine direction and magnitude of $\mathbf{R}$. Past investigations found that the collective electron phase $\left(\mathbf{p}_{e 1}+\mathbf{p}_{e 2}\right) \cdot \mathbf{R}$ plays an important role in the double-slit interference [5-7], instead of the single-electron phase $\mathbf{p}_{e} \cdot \mathbf{R}$. Recently, Waitz et al. [8] showed that it is also possible to retrieve $\mathbf{R}$ and observe double-slit interference for the dissociative single ionization channel.

In 2020, an experiment [9] on double ionization of $\mathrm{H}_{2}$ by a single, circularly polarized photon of $800 \mathrm{eV}$ energy confirmed the expectation of an extra phase shift of $-\mathbf{k}_{\gamma}$. $\mathbf{R}$ to the interference pattern of molecular photoionization [10], where $\mathbf{k}_{\gamma}$ is the wave vector of the light field. This phase shift directly connects to the travel time $\Delta \tau$ of the light passing through the molecule. Note that the bound state phase $I_{p} \Delta \tau$ was neglected in Ref. [9] due to its negligible effects in physical observables, which of course should be included when one considers the equivalence between these two physical pictures.

In the experiment, the phase difference has been extracted by measuring the shift of the interference maximum of the

\footnotetext{
*liangyou.peng@pku.edu.cn
}

single electron angular distribution while only the extreme energy partition $\left(E_{1} /\left(E_{1}+E_{2}\right)>96 \%\right)$ was taken into account in the analysis. So far, the implications of electron-electron correlation on this intriguing effect remain unexplored.

We address this issue in the present work by numerically solving the full-dimensional two-electron time-dependent Schrödinger equation (TDSE) of $\mathrm{H}_{2}$ with the inclusion of the leading order nondipole terms in the interaction Hamiltonian, which is given in the Poincaré gauge by

$$
H_{\mathrm{int}}=\sum_{i=1,2} \mathbf{r}_{i} \cdot \mathbf{E}(t)-\frac{\hat{\mathbf{k}}_{\gamma} \cdot \mathbf{r}_{i}}{2 c} \mathbf{r}_{i} \cdot \dot{\mathbf{E}}(t)+\frac{\left[\hat{\mathbf{k}}_{\gamma} \times \mathbf{E}(t)\right]}{2 c} \cdot \mathbf{l}_{i},
$$

where $\mathbf{E}(t)$ is the electric field at the origin of the coordinates, $\mathbf{r}_{i}$ and $\mathbf{l}_{i}$ represents the position and angular momentum operator of the $i$-th electron, respectively. Atomic units are used throughout the paper unless specified otherwise. With the internuclear distance $R$ fixed at $R_{e}=1.4$ a.u., we expand the electronic wave function in prolate spheroidal coordinates $(\xi, \eta, \phi)$ with a production basis of the finite element discrete variable representation [11] and spherical harmonics. After discretization, one can search for the ground state $|0\rangle$ of the field free Hamiltonian $\mathrm{H}_{0}$ of $\mathrm{H}_{2}$ with the restarted Lanczos scheme and then propagate $\left(\mathrm{i} \partial_{t}-H_{0}\right)|\psi\rangle=H_{\text {int }}|0\rangle$ in time with the short-time Arnoldi propagator [12]. Specific forms of each term in the Hamiltonian can be found in Refs. [13] and [14]. It is worth to mention that, unlike the atomic case [15], the last term in Eq. (1), i.e., the magnetic dipole term, does not vanish and should be included in the computation, since 

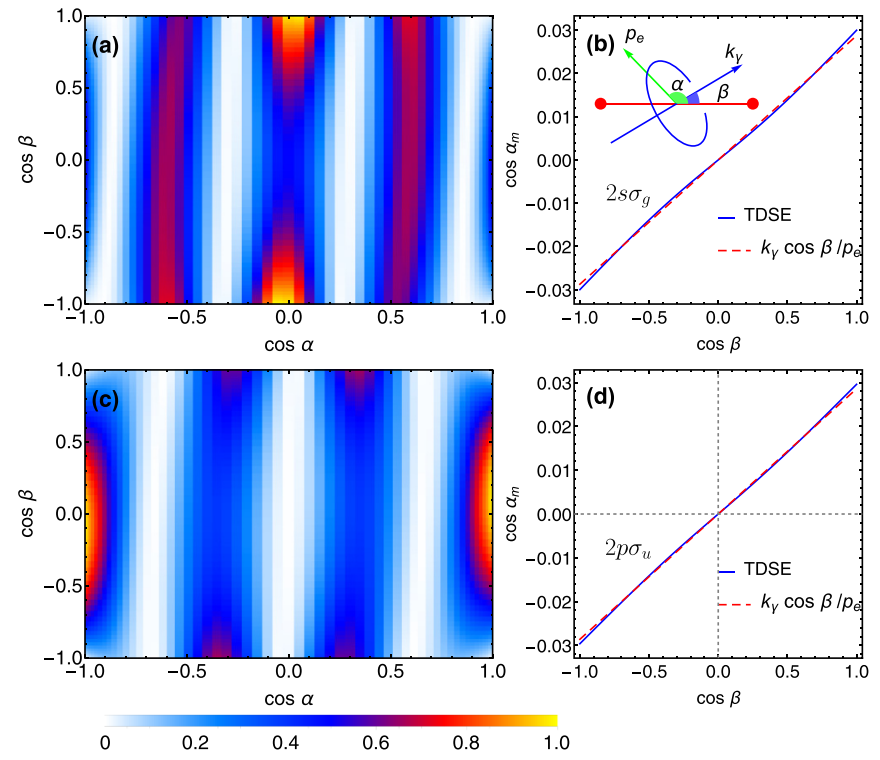

FIG. 1. Single ionization of $\mathrm{H}_{2}$ by one $800 \mathrm{eV}$ circularly polarized photon. (a), (c) Electron angular distribution in the molecular frame of reference as a function of $\cos \beta$ where the remaining bound electron stays in the $2 s \sigma_{g}$ (a) or $2 p \sigma_{u}$ (c) state. (b) Position of zeroth order interference maximum $\cos \alpha_{m}$ as a function of $\cos \beta$ for data shown in (a). (d) Position of zeroth order interference minimum $\cos \alpha_{m}$ as a function of $\cos \beta$ for data shown in (c). The solid blue lines in (b) and (d) represent results from TDSE calculation, and the dashed red lines are the model prediction $\cos \alpha_{m}=k_{\gamma} \cos \beta / p_{e}$. The inset in (b) shows the configuration of molecular axis, wave vector, and polarization of light, and the electron emission direction.

the total angular momentum is not a good quantum number for a molecule without the central symmetry.

To simulate the experiment, we apply a 10-cycle circular polarization pulse with the $\cos ^{2}$ envelope at the center frequency of $\omega=800 \mathrm{eV} \approx 29.4$ a.u. to ionize the molecule. After another 2-cycle's field-free propagation at the end of the pulse, the wave function is projected into the production of the single-electron continuum of the diatomic molecule. The inset of Fig. 1(b) shows the configuration of the molecular axis, the electron emission direction, the light wave vector, and the polarization of the light. $\alpha$ and $\beta$ denote the polar angles of the electron emission direction and the light wave vector, both with respect to the molecular axis. We set the time step $\Delta t=0.005$ a.u., the radius of simulation volume $\xi_{\max } R_{e} / 2 \approx 60$ a.u., and the average grid spacing $\Delta \xi R_{e} / 2 \approx$ 0.3 a.u. The maximum index of spherical harmonics is chosen to be $l_{\max }=11$ and $m_{\max }=3$. The numerical convergence has been guaranteed for all the numerical parameters.

Let us first discuss the single ionization channel. The final wave function is projected onto the direct production of bound states of $\mathrm{H}_{2}^{+}$and the continuum state of half charged $\mathrm{H}_{2}^{+}$ for the consideration of the screening effect. The calculated one-photon single ionization cross-sections for the dominant three channels are $\sigma\left(1 s \sigma_{g}\right)=62.2 \mathrm{barn}, \sigma\left(2 s \sigma_{g}\right)=2.5 \mathrm{barn}$ and $\sigma\left(2 p \sigma_{u}\right)=1.2$ barn. Cross sections for all other single ionization channels are less than 1 barn and thus can be neglected. We choose $2 s \sigma_{g}$ and $2 p \sigma_{u}$ states for the analysis since they are two lowest dissociative electronic states. Figure 1(a) shows the electron angular distribution as function of $\cos \beta$ for the $2 s \sigma_{g}$ channel. The two-center interference is clearly visible as the electron yield is proportional to $\cos \left(p_{e} R \cos \alpha\right)$. However, as the angle between $\mathbf{k}_{\gamma}$ and $\mathbf{R}$ changes, the interference maximum will also shift leftward or rightward because of the spatially dependent light phase. Accordingly, the electron yield is proportional to $\cos \left(p_{e} R \cos \alpha-k_{\gamma} R \cos \beta\right)$ and one can conclude that the zeroth order interference maximum should shift to

$$
\cos \alpha_{m}=\frac{k_{\gamma}}{p_{e}} \cos \beta .
$$

In Fig. 1(b), we plot $\cos \alpha_{m}$ as a function of $\cos \beta$ and compare it with the simple prediction of Eq. (2). One finds that there is a nearly perfect agreement between the results of this simple model and our ab initio calculations. The small discrepancies (including the tiny oscillation of the TDSE results) are tolerable, if one considers the simplification of the model and the fact that only the first-order nondipole terms are taken into account in the calculations. In Fig. 1(c), a phase shift of $\pi$ is apparent for the $2 p \sigma_{u}$ channel (relative to the $2 s \sigma_{g}$ channel), which was also observed in recent experiments [8]. The reason is that the wave function of the $2 p \sigma_{u}$ state has the form of $\mid$ left $\rangle-\mid$ right $\rangle$, which has a phase difference of $\pi$ between the two nuclei. In this case, we take the zeroth order interference minimum and plot it in Fig. 1(d). Again, a nearly perfect agreement can be seen between the results of the model and the calculations.

Now, let us consider the double ionization channel. Here, the electron momentum distribution is evaluated by projecting the final wave function onto the direct product of continuum states of $\mathrm{H}_{2}^{+}$. The total cross-section for one-photon double ionization is 0.75 barn. In Fig. 2(a), we present the calculated energy partition differential cross section $d \sigma / d r$ between the two electrons. Due to the exchange symmetry, we only plot the region of the energy partition rate $r \equiv E_{1} /\left(E_{1}+E_{2}\right)>0.5$. For the considered photon energy of $800 \mathrm{eV}$, most of the ionization events happen through the shake-off mechanism where one electron takes most of the available kinetic energy [16]. In order to compare our calculations to the experimental results in Ref. [9], we integrate over the slow electron by

$$
P_{\text {single }}\left(\cos \alpha_{1}\right) \equiv \int P_{\text {joint }}\left(\cos \alpha_{1}, \cos \alpha_{2}\right) d \cos \alpha_{2}
$$

In Fig. 2(b), we show the angular distribution of the fast electron with $r>96 \%$ as indicated by the shadow area in Fig. 2(a). The fringes are tilted like in Fig. 1(a) but the visibility is considerably worse. In Fig. 2(c), we plot $\cos \alpha_{m}$ as a function of $\cos \beta$ for three different energy partition rates $r=99 \%, 97 \%, 96 \%$, and compare them with the prediction of Eq. (2) and the experimental results from Ref. [9]. Here, the agreement between the model prediction and the numerical results is only fair since the slopes for the latter are larger and sensitively dependent on the energy partition rate $r$. In Ref. [9], the larger slope of the experimental results $(r>96 \%)$ compared to the model prediction could not be explained. Our finding that the slope depends on the energy partition rate might resolve this issue, but the large error bars still make it hard to draw a definite conclusion. 

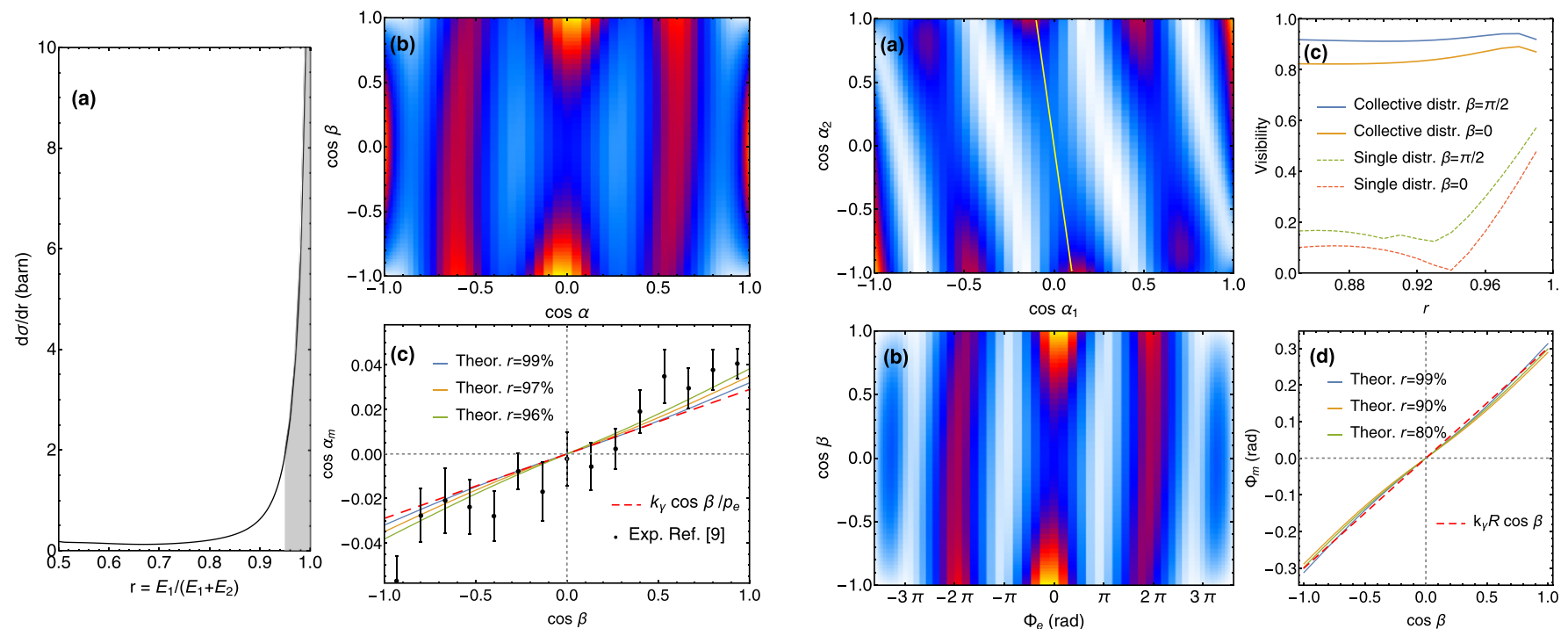

FIG. 2. Double ionization of $\mathrm{H}_{2}$ by one $800 \mathrm{eV}$ circularly polarized photon. (a) Energy partition differential cross section after averaging over the angle $\beta$ between light wave vector $\mathbf{k}_{\gamma}$ and molecular axis $\mathbf{R}$. The shaded area indicates where the energy partition rate $r$ is larger than 96\%, which has been used for analysis in Ref. [9]. (b) Same as Fig. 1 (a), but for double ionization where the fast electron carries more than $96 \%$ of the kinetic energy. (c) Same as Fig. 1 (b), but for double ionization. The three solid lines (blue, yellow, and green) present TDSE results with energy partition rates of $99 \%, 97 \%$ and $96 \%$, respectively. The dashed red line shows the model prediction $\cos \alpha_{m}=k_{\gamma} \cos \beta / p_{e}$, where $p_{e}=\sqrt{2\left(\omega-I_{p}\right)}$ (approximating that one electron takes the whole kinetic energy). The black dots with error bars show the experimental results from Ref. [9] for comparison.

Next, we further investigate the effect of electron correlation. In Fig. 3(a), we plot the joint angular distribution $P_{\text {joint }}\left(\cos \alpha_{1}, \cos \alpha_{2}\right)$ at $r=99 \%$ and $\cos \beta=0$. There is a clear correlation between the emission angles of the two electrons. In fact, the tilted fringes follow the relationship $\Phi_{e} \equiv$ $p_{e 1} R \cos \alpha_{1}+p_{e 2} R \cos \alpha_{2}=$ Const. Similar phenomena for one-photon double ionization of $\mathrm{H}_{2}$ were observed previously in the near-equal energy sharing region with a photon energy of $400 \mathrm{eV}$ [7]. It indicates that these two electrons can be regarded as an entangled pair emitted from the same nucleus, no matter whether $r \approx 0.5$ or $r \approx 1$. The reason why this correlation is visible to the naked eye even at the extreme energy partition rate in Fig. 3(a) is because that the electron momentum is proportional to the square root of the kinetic energy. For example, the slope is $p_{e 2} / p_{e 1}=\sqrt{E_{2} / E_{1}} \approx 0.1$ at $r=99 \%$, which is by far not an ignorable quantity.

As the interference fringes are dependent on the emission direction of the slow electron, one can conclude that the fringe visibility will be largely suppressed if one only integrates over the slow electron, as done so in Fig. 2 and Ref. [9]. For a quantitative study, we define the fringe visibility as $V \equiv$ $\left(Y_{\max }-Y_{\min }\right) /\left(Y_{\max }+Y_{\min }\right)$, where $Y_{\max }$ is the yield of the zeroth order interference maximum, and $Y_{\min }$ is the average yield of the -1 and +1 order minimum. In Fig. 3(c), the two lines near the bottom show the dependence of the fringe visibility on energy partition rate $r$ at $\beta=0$ and $\pi$, respectively. One
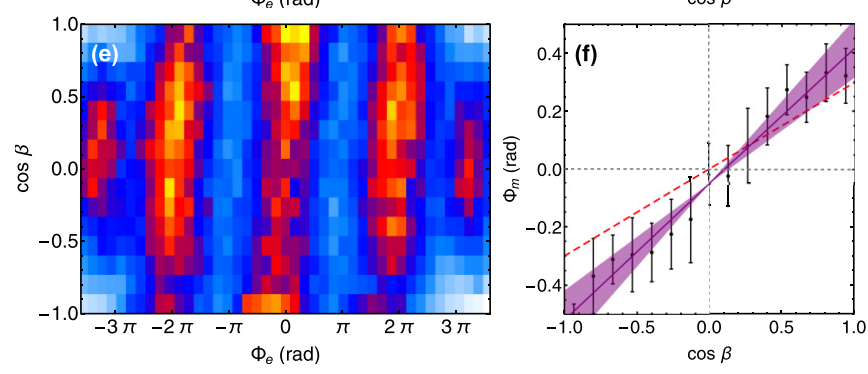

FIG. 3. (a) Joint angular distribution with $r=99 \%$ for perpendicular orientation of light wave vector and molecular axis $(\beta=\pi / 2)$. The yellow line indicates $p_{e 1} \cos \alpha_{1}+p_{e 2} \cos \alpha_{2}=0$. (b) $P_{\text {collect }}\left(\Phi_{e}\right)$ as function of $\cos \beta$ for $r=99 \%$ (see text for definition). (c) Fringe visibilities, from top to bottom: $\beta=\pi / 2$ for $P_{\text {collect }}\left(\Phi_{e}\right), \beta=0$ for $P_{\text {collect }}\left(\Phi_{e}\right), \beta=\pi / 2$ for $P_{\text {single }}\left(\cos \alpha_{1}\right), \beta=0$ for $P_{\text {single }}\left(\cos \alpha_{1}\right)$. (d) Position of zeroth order interference maximum $\Phi_{m}$ as a function of $\cos \beta$. The three solid lines with (blue, yellow, and green) present TDSE results with energy partition rates of $99 \%$, $90 \%$ and $80 \%$, respectively. The dashed red line represents the simple model $\Phi_{m}=k_{\gamma} R \cos \beta$. (e) Experimental results for comparison to (b). Unlike Ref. [9], all the double ionization events are taken into account here. (f) The black dots present the mean of a Gaussian fit to the zeroth order peak for each $\cos \beta$ in (e) and the error bars indicate the $95 \%$ confidence intervals. The shaded purple region shows the respective linear fit. The dashed red line is the same as in (d).

finds that the visibility quickly decreases to a relatively low value, which means that fringes in $P_{\text {single }}$ are nearly washed out and experimentally invisible. Furthermore, the center of the zeroth order interference maximum of $P_{\text {single }}\left(\cos \alpha_{1}\right)$ may also be shifted due to the asymmetric distribution along the fringe.

To overcome the above problem, one can integrate along the contour of the collective electron phase $\Phi_{e}$ and thus has

$$
\begin{aligned}
P_{\text {collect }}\left(\Phi_{e}\right) \equiv & \int P_{\text {joint }}\left(\cos \alpha_{1}, \cos \alpha_{2}\right) \delta\left(\Phi_{e}-p_{e 1} R \cos \alpha_{1}\right. \\
& \left.-p_{e 2} R \cos \alpha_{2}\right) d \cos \alpha_{1} d \cos \alpha_{2} .
\end{aligned}
$$

We plot $P_{\text {collect }}\left(\Phi_{e}\right)$ as a function of $\cos \beta$ in Fig. 3(b) at $r=99 \%$. Obviously, the fringes are more visible than those in Fig. 2(b). In Fig. 3(c), the two lines near the top show the 
fringe visibility for $P_{\text {collect }}\left(\Phi_{e}\right)$ at $\beta=0$ and $\pi$, respectively, which always keep a relatively large value for all $r$. Now, one can search for the position of zeroth order interference maximum $\Phi_{m}$ as a function of $\cos \beta$. As can be seen in Fig. 3(d), our $a b$ initio results perfectly agree with the model prediction for all the three chosen $r$. Therefore, the maximum appears when the collective electron phase $\Phi_{e}$ exactly cancels out the light phase $-k_{\gamma} R \cos \beta$, independent of the energy partition between the two electrons.

The fact that both electrons emit from the same nucleus implicitly resolves a tricky problem. If the electrons were emitted from two different nuclei, the phase of one twoelectron interference channel would be $\left(\boldsymbol{p}_{e 1} \cdot \boldsymbol{r}_{L}+\boldsymbol{p}_{e 2} \cdot \boldsymbol{r}_{R}\right)$ [5]. Accordingly, it would be unclear how to determine the extra phase shift due to the spatially dependent light field. In the shake-off picture, one could assign the light phase to the nucleus from which the fast electron emits. But with a varying energy partition rate, this assignment becomes ambiguous. This problem does not arise in the present case of a large photon as both electrons actually emit from the same nucleus.

From the discussion above, we propose that one can collect all the double ionization events measured in experiments and plot them as in Fig. 3(b). In doing so, one can improve the fringe visibility and reduce the statistical fluctuation. Accordingly, we plot the corresponding experimental data in Fig. 3(e). Note that these results are generated from the same experimental dataset as Ref. [9], where further details can be found. Indeed, we find that the interference patterns are now much more clear than those in Ref. [9]. The fitted peak positions are now plotted in Fig. 3(f) as a function of $\cos \beta$ with smaller error bars and more regular behaviors. Nevertheless, we find that the experimental measurements still deviate from the results of the model prediction and our TDSE calculations.

We have considered a further possible reasons for this deviation. One may wonder if the molecular axis measured experimentally really reflects the axis at the moment the ionization occurs. In the experiment, the molecular axis is determined by the direction of the relative momentum of the two protons, with the assumption that the momentum mainly comes from the Coulomb repulsion with $p_{\text {rep }} \approx \sqrt{m_{p} / R} \approx 36$ a.u.. For the double-slit interference considered, electrons ionized from one nucleus interfere with electrons ionized from the other one, resulting in a recoil momentum from the electron of $p_{\text {rec }} \approx \sqrt{2 \omega} \approx 8$ a.u.. There seems to be an unignorable rotation $p_{\text {rec }} / p_{\text {rep }} \approx 0.22$ rad to the molecular axis for the case of $\alpha \sim \pi / 2$. By taking the recoil momentum into account, we treat the nuclear motion quantum mechanically within the Born-Oppenheimer approximation [17]. However, we do not find significant differences in the results from the models with and without consideration of the recoil momentum. One may interpret it as the cancellation between the two opposite directions of rotation. The two directions of the rotation do not provide the which-path-information for the rotational ground state we consider here [18]. In addition, the temperature of the gas target is not high enough to excite the molecular to excited rotational states. At this point, we may assume that the deviation between experiment and model prediction is mainly due to a systematic experimental error.

In conclusion, we have investigated the modification of the double-slit interference in one-photon ionization of $\mathrm{H}_{2}$ due to a spatially dependent light phase. For the single ionization, we find excellent agreement between TDSE results and the simple model prediction of light with spatial phase dependence launching two coherent electron waves near different nuclei of the molecule. For the double ionization, one has to integrate along the contour of the collective electron phase to achieve an agreement between the model prediction and the TDSE results for all the double ionization events. Otherwise, if one just integrates over the slow electron, the result will sensitively depend on the energy partition between the two electrons. Nevertheless, further investigations are needed to account for the existing deviation between the theoretical and experimental results.

The authors are grateful to R. Dörner for a number of insightful discussions via email correspondences. The theoretical work is supported by the National Natural Science Foundation of China (NSFC) under Grants No. 11961131008 and No. 11725416 and by the National Key R\&D Program of China under Grant No. 2018YFA0306302. We are grateful to D. Trabert, K. Fehre, N. Strenger, A. Pier, L. Kaiser, M. Kircher, M. Weller, S. Eckart, L. Schmidt, F. Trinter, T. Jahnke, and M. Schöffler for their significant contributions to the experiment and the data analysis. The experimental work was supported financially by DFG and BMBF. We acknowledge DESY (Hamburg, Germany), a member of the Helmholtz Association HGF, for the provision of experimental facilities. Parts of this research were carried out at PETRA III and we would like to thank the staff for their excellent support during the beam time. S.G. acknowledges support from Deutsche Forschungsgemeinschaft via Sonderforschungsbereich 1319 (ELCH).
[1] D. Akoury, K. Kreidi, T. Jahnke, T. Weber, A. Staudte, M. Schöffler, N. Neumann, J. Titze, L. P. H. Schmidt, A. Czasch, O. Jagutzki, R. A. C. Fraga, R. E. Grisenti, R. D. Muiño, N. A. Cherepkov, S. K. Semenov, P. Ranitovic, C. L. Cocke, T. Osipov, H. Adaniya et al., Science 318, 949 (2007).

[2] H. D. Cohen and U. Fano, Phys. Rev. 150, 30 (1966).

[3] J. Fernández, O. Fojón, A. Palacios, and F. Martín, Phys. Rev. Lett. 98, 043005 (2007).

[4] S. E. Canton, E. Plesiat, J. D. Bozek, B. S. Rude, P. Decleva, and F. Martin, Proc. Natl. Acad. Sci. USA 108, 7302 (2011).
[5] K. Kreidi, D. Akoury, T. Jahnke, T. Weber, A. Staudte, M. Schöffler, N. Neumann, J. Titze, L. P. H. Schmidt, A. Czasch, O. Jagutzki, R. A. Costa Fraga, R. E. Grisenti, M. Smolarski, P. Ranitovic, C. L. Cocke, T. Osipov, H. Adaniya, J. C. Thompson, M. H. Prior et al., Phys. Rev. Lett. 100, 133005 (2008).

[6] D. A. Horner, S. Miyabe, T. N. Rescigno, C. W. McCurdy, F. Morales, and F. Martín, Phys. Rev. Lett. 101, 183002 (2008).

[7] M. Waitz, D. Metz, J. Lower, C. Schober, M. Keiling, M. Pitzer, K. Mertens, M. Martins, J. Viefhaus, S. Klumpp, T. Weber, H. Schmidt-Böcking, L. P. H. Schmidt, F. Morales, S. Miyabe, 
T. N. Rescigno, C. W. McCurdy, F. Martín, J. B. Williams, M. S. Schöffler et al., and Phys. Rev. Lett. 117, 083002 (2016).

[8] M. Waitz, R. Y. Bello, D. Metz, J. Lower, F. Trinter, C. Schober, M. Keiling, U. Lenz, M. Pitzer, K. Mertens, M. Martins, J. Viefhaus, S. Klumpp, T. Weber, L. P. H. Schmidt, J. B. Williams, M. S. Schöffler, V. V. Serov, A. S. Kheifets, L. Argenti et al., Nat. Commun. 8, 2266 (2017).

[9] S. Grundmann, D. Trabert, K. Fehre, N. Strenger, A. Pier, L. Kaiser, M. Kircher, M. Weller, S. Eckart, L. P. H. Schmidt, F. Trinter, T. Jahnke, M. S. Schöffler, and R. Dörner, Science 370, 339 (2020).

[10] G. L. Yudin, S. Chelkowski, and A. D. Bandrauk, J. Phys. B 39, L17 (2005).
[11] T. N. Rescigno and C. W. McCurdy, Phys. Rev. A 62, 032706 (2000).

[12] H. Liang, X.-R. Xiao, Q. Gong, and L.-Y. Peng, J. Phys. B: At. Mol. Opt. Phys. 50, 174002 (2017).

[13] L. Tao, C. W. McCurdy, and T. N. Rescigno, Phys. Rev. A 82, 023423 (2010).

[14] H. Liang, M.-X. Wang, X.-R. Xiao, Q. Gong, and L.-Y. Peng, Phys. Rev. A 98, 063413 (2018).

[15] S.-G. Chen, W.-C. Jiang, S. Grundmann, F. Trinter, M. S. Schöffler, T. Jahnke, R. Dörner, H. Liang, M.-X. Wang, L.-Y. Peng, and Q. Gong, Phys. Rev. Lett. 124, 043201 (2020).

[16] C. Siedschlag and T. Pattard, J. Phys. B 38, 2297 (2005).

[17] V. V. Serov and A. S. Kheifets, J. Phys. B 47, 115006 (2014).

[18] K. Ueda, J. Phys.: Conf. Ser. 212, 012033 (2010). 